\title{
Transverse Myelitis Caused by Varicella Zoster: Case Reports
}

\author{
Sema Yýlmaz ${ }^{1}$, Hamide Kart Köseoðlü ${ }^{2}$ and Eftal Yücel $^{2}$ \\ ${ }^{1}$ Baskent University Konya Medical and Research Center, Division of Rheumatology, Konya; ${ }^{2}$ Baskent University Faculty of Medicine, \\ Division of Rheumatology, Ankara; Turkey
}

\begin{abstract}
Transverse myelitis is a group of disorders characterized by focal inflammation of the spinal cord and results in loss of motor and sensory function below the level of injury. Occurrence of this condition during or following varicella infection is uncommon. This report describes two cases of transverse myelitis caused by varicella zoster. Key-Words: Varicella zoster, infection, transverse myelitis.
\end{abstract}

Varicella zoster virus (VZV) infection causes a variety of neurologic complications, including post-herpetic neuralgia, polyradiculoneuritis, transverse myelitis, vasculopathy, aseptic menengitis, leukoencephalopathy, dorsal root or cranial nerve ganglionitis, ventriculitis, necrotising angiitis and meningoencephalitis [1,2]. Transverse myelitis has been rarely associated with VZV infection. Onset is acute and occurs shortly after typical cutaneous rash in a dermatomal distribution with the development of paraparesis, sensory loss and sphincter dysfunction [3]. No diagnostic test is completely accurate, since VZV can not usually be isolated from blood or cerebrospinal fluid (CSF) in VZV myelitis. Treatment involves high doses of both steroids and acyclovir. Recovery is usually spontaneous, though fatal cases have been reported [4].

We describe two cases of transverse myelitis during and following VZV infection that were proven serologically and neurologically.

\section{Case 1}

A 48-year-old woman was presented to our clinic in April 2003 with the complaints of right leg weakness and urinary incontinence. Two months before, she had experienced a painful vesicular eruption on the lateral side of her left breast. Herpes zoster was diagnosed, and she was treated with a 10day course of oral acyclovir ( $800 \mathrm{mg}$ five times daily). In March 2003, she had been admitted to another hospital with paresthesia and weakness in both legs. Magnetic resonance imaging (MRI) of the spinal cord demonstrated lesions of high-signal intensity at C1-2 and T2-3 levels. The lesions appeared to be masses, therefore subtotal laminectomy was performed at T1-3. Histopathologic examination showed perivascular lymphocyte infiltration and reactive gliosis.

On admission to our center in April, neurologic examination revealed normal levels of consciousness, cooperation, orientation and showed that all cranial nerves were intact. The patient exhibited 1/5 lower extremity weakness on the right and 3/5 on the left, diminished sensation to pain and

Received on 6 September 2006; revised 12 December 2006.

Address for correspondence: Dr. Sema Yýlmaz. Vatan cad. Devrim sitesi B/Blok. Kat:2 No:29/4. Zip code: 42040 Selçuklu, Konya/Turkey. Phone: + 90-332-3200484. E-mail: drsemayilmaz@hotmail.com

The Brazilian Journal of Infectious Diseases 2007;11(1):179-181. (C) 2007 by The Brazilian Journal of Infectious Diseases and Contexto Publishing. All rights reserved. light touch below the level of $\mathrm{T} 5$ dermatome, and marked loss of vibratory sensation and proprioception in both legs. Deep tendon reflexes were normal in the upper extremities, but decreased in the lower extremities. There was no nuchal rigidity.

Laboratory findings for complete blood cell count, liver enzymes, BUN, creatinine, C-reactive protein, erythrocyte sedimentation rate and urinalysis were normal. Serological tests for hepatitis B and C, brucellosis, cytomegalovirus, herpes simplex virus, Epstein-Barr virus, PPD and human immunodeficiency virus were all negative. The serum was positive for anti-VZV antibodies. CSF was negative for antiVZV antibodies.Tests for anti-nuclear antibodies, anti-double stranded DNA, and cytoplasmic and perinuclear types of antineutrophil cytoplasmic antibodies were also negative. Cranial MRI examination was normal. Cervical MRI demonstrated lesions of high signal intensity at C2 and C5-C6 levels, and the appearance was consistent with areas of focal edema. Thoracic MRI showed the laminectomy defects, fluid collection at T1-T4 and enhancement was seen after administration of gadolinium. Lumbosacral MRI revealed edema around the interspinous ligaments at L4-L5 and L5-S1.

The patient was diagnosed with transverse myelitis, and treatment was started with oral methylprednisolone $1 \mathrm{mg} / \mathrm{kg} /$ day was initiated. This was continued for two weeks and was then tapered by $4 \mathrm{mg}$ each week until $10 \mathrm{mg} /$ day. Gabapentin $2,000 \mathrm{mg} /$ day, amitriptyline $25 \mathrm{mg} /$ day and citalopram $20 \mathrm{mg} /$ day were also prescribed. After 3 months, she was able to walk supported by canes, and despite mild leg weakness, had normal sensation in her legs and normal sphincter function.

\section{Case 2}

A 52-year-old woman was presented to our clinic in March 2003 with low back pain, paresthesia in both legs. In June of 2001, she had presented with leg pain and a painful itchy vesicular eruption in her lumbosacral area. Herpes zoster was diagnosed, and she was treated with 10 days of oral acyclovir $800 \mathrm{mg}$ five times daily. During this treatment period, she developed paresthesia and weakness in both legs, urinary and fecal incontinence. She was hospitalized. Spinal cord MRI at the time showed intramedullary lesions of high signal intensity between the T2-T3 and T10 levels with gadolinium enhancement, findings which are consistent with myelitis. Antibodies to VZV were detected in serum but not in CSF. The patient was placed on a treatment regimen for transverse 
myelitis, and was transferred to the rehabilitation unit in another hospital. By the end of therapy, she had improved dramatically.

Initial neurologic examination on admission in March 2003 showed normal levels of consciousness, cooperation, orientation, as well as intact cranial nerves and normal cerebellar function. Motor function in the upper and lower extremities was normal, but diminished sensation to pain and light touch was noted below the T12 dermatome. Vibratory sensation and proprioception were diminished in the patients' left lower leg. Deep tendon reflexes were normal in the upper extremities, but decreased in the lower extremities. There was no nuchal rigidity. Ophtalmoscopic examination revealed nothing remarkable.

Laboratory results for complete blood cell count, liver enzymes, BUN, creatinine, C-reactive protein, erythrocyte sedimentation rate and urinalysis were normal. Serological tests for brucellosis, hepatitis B and C, herpes simplex virus, cytomegalovirus, Epstein-Barr virus, PPD and human immunodeficiency virus were negative. Serum was positive for antibodies to VZV. CSF was negative for anti-VZV antibodies. Testing for antinuclear antibodies, anticardiolipin antibodies immunoglobulin $\mathrm{G}$ and immunoglobulin $\mathrm{M}$, cytoplasmic and perinuclear types of anti-neutrophil cytoplasmic antibodies, cryoglobulin and complement 3 and 4 showed nothing abnormal. Cranial and thoracic MRI examinations were normal; however, lumbosacral MRI showed stenosis of the neural foramen at L4-L5. Electromyography showed mild loss of motor unit action potentials.

The patient was treated with oral methylprednisolone 20 $\mathrm{mg} /$ day for 3 weeks and was then tapered by $4 \mathrm{mg}$ every week until $4 \mathrm{mg} /$ day. She was also prescribed amitriptyline $50 \mathrm{mg} /$ day and gabapentin $600 \mathrm{mg} /$ day. After 10 weeks, she had normal sensation in the legs and mild low back pain.

\section{Discussion}

Transverse myelitis is an unusual inflammatory disease involving the entire thickness of the spinal cord. It results in loss of sensory and motor function below the level of injury. The condition may be acute, developing over a few hours or several days, or subacute, developing over 1 to 2 weeks. The critical factor seems to be abnormal immune response to an infection rather than the direct effect of an infectious agent. Transverse myelitis may be an isolated entity or may occur in the context of multifocal or even multisystemic disease. Transverse myelitis is known to occur on a background of viral diseases, vaccinations, systemic lupus erythematosus, vasculitis, multipl sclerosis, heroin abuse and trauma [5]. Approximately 25\%-40\% cases of transverse myelitis are caused by viral infections with herpes viruses and poliovirus. Varicella zoster infection is not a common cause of transverse myelitis in immunologically normal patients. The typical clinical picture of transverse myelitis is involvement of both motor and sensory tracts at one level. The spectrum of clinical outcomes in VZV myelitis ranges from spontaneous recovery to ascending progression and death [6].Patients with rapid progression and flaccidity below the level of the lesion have the poorest prognosis.

The frequency of transverse myelitis during or after varicella infection is $0.3 \%$ [7]. The characteristic symptoms are typically bilateral sensory deficit at a given level, paraparesis, quadriparesis, motor weakness and abnormal bladder and rectal function. In most cases, transverse myelitis caused by VZV is diagnosed based on detection of typical vesicular lesions in dermatomal distribution that are associated with signs and symptoms suggestive of transverse myelitis. However, transverse myelitis associated with herpes zoster have been described in the absence of typical skin lesions [8].

The pathogenesis of neurological complications associated with VZV infection is unclear. An allergic and vascular mechanisms have been suggested for some of these neurological complications that occur after both primary varicella infection and herpes zoster [9]. In idiopathic acute transverse myelitis, there is an intraparenchymal or perivascular cellular influx into the spinal cord, resulting in the breakdown of the blood-brain barrier and variable demyelination and neuronal injury. However, the pathogenesis of VZV myelitis has been thought as a direct viral invasion, because VZV particles were found in glial cells, and the virus was isolated from the spinal cord of patients with zoster myelitis. In a detailed report that included post mortem examination of the spinal cord, Hogan et al. [10] presented evidence of direct invasion of VZV in a patient with transverse myelitis associated with herpes zoster. Demonstration of the VZV antigen in CSF cells by immunofluorescence or isolation of VZV from the CSF is a confirmative evidence for viral central nervous system infection but it is rarely successful [11].

In both of our cases, transverse myelitis related to varicella infection was diagnosed on the basis of MRI findings, and on the development of motor weakness, paraparesis, rectal and bladder dysfunction following appearance of a rash. Both patients' serum contained anti-VZV antibodies. We didn't detect VZV antibodies in CSF, because VZV can not usually be isolated from CSF in VZV myelitis.

Diagnosing VZV myelitis can be challenging. Physicians must maintain a high index of suspicion in atypical cases. It is important to rule out spinal cord compression due to epidural abscess or tumor, intrinsic bacterial or fungal infections of the spinal cord, and treatable vascular diseases. Spinal tap,computerized tomography, MRI, myelography and blood tests may help narrow the diagnostic possibilities.

There are no established treatment regimens for transverse myelitis as a complication of VZV infection. Some researchers recommend high doses of acyclovir and steroid. We treated two patients with oral acyclovir, methylprednisolone, gabapentin and amiptriptylin. Both women improved within 10 weeks of the start of therapy, and their complaints were markedly decreased after treatment was completed. At 1 year of follow-up, both patients had fully recovered. 


\section{References}

1. Chang C.M., Woo E., Yu Y.L., et al. Herpes zoster and its neurological complications. Post-grad Med J 1987;63:85-9.

2. Bucci F.A., Schwartz R.A. Neurologic complications of herpes zoster. Am Fam Physician 1988;37:185-92.

3. Gilden D.H., Mahalingam R., Dueland A.N., Cohrs R. Herpes zoster: pathogenesis and latency. In: Melnick J.N. eds. Progress in medical virology. Basel, Karger, 1992:19-75.

4. Çelik Y., Tabak F., Mert A., Aktuðlu T. Transverse myelitis caused by varicella. Clin Neurol and Neurosurg 2001;103:260-1.

5. Jubelt B., Miller J. Viral infections. In: Rowland Lewis P et al,.eds. Merritt's Neurology. Philadelphia: WB Saunders, 2001:152.

6. Hýrai T., Korogi Y., Hamatake S., et al. Case report: Varicella-zoster virus myelitis-serial MR findings. Br J Radiology 1996;69:1187-90.
7. de Silva S.M., Mark A.S., Gilden D.H., et al. Zoster myelitis: improvement with antiviral therapy in two cases. Neurology 1996;47:929-31.

8. Mayo D., Booss J. Varicella zoster-associated neurologic disease without skin lesions. Arch Neurol 1989;46: 313-5.

9. Gelb L.D. Varicella-zoster virus. In: Fields BN, Knipe DM, Chanock RM. et al.,eds.Virology. New York: Raven Press, 1990:2011-54.

10. Hogan E.L., Krigman M.R. Herpes zoster myelitis. Evidence for viral invasion of spinal cord. Arch Neurol 1973;29:30913.

11. Hwang Y.M., Lee B.I., Chung J.W., et al. A case of herpes zoster myelitis: Positive magnetic resonance imaging finding. Eur Neurol 1991;131:164-7. 